\title{
Evaluation of tungsten isotopes in the fast neutron range including cross section covariance estimation
}

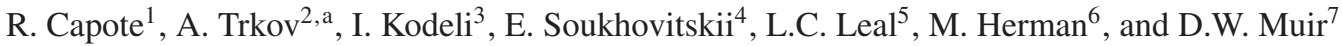 \\ 1 Nuclear Data Section, International Atomic Energy Agency, 1400 Vienna, Austria \\ 2 Jozef Stefan Institute, Ljubljana, Slovenia \\ 3 International Atomic Energy Agency representative at the OECD/NEA Data Bank, 92130 Issy-les-Moulineaux, France \\ 4 Joint Institute for Energy and Nuclear Research, 220109 Minsk-Sosny, Belarus \\ 5 Oak Ridge National Laboratory, P.O. Box 2008, Oak Ridge, TN 37831-6356, USA \\ 6 National Nuclear Data Center, Brookhaven National Laboratory, Upton, NY, USA \\ 7 International Atomic Energy Agency (Consultant), P.O. Box 452, Kittery, Maine, USA
}

\begin{abstract}
New evaluations for the tungsten isotopes ${ }^{180,182,183,184,186} \mathrm{~W}$ in the neutron energy range up to $60 \mathrm{MeV}$ were produced. In the resonance range only minor adjustments to the resonance parameters were made due to a lack of adequate experimental data. Evaluations in the fast energy region were based on nuclear model calculations using the EMPIRE-2.19 code. Recently derived dispersive coupled-channel optical model potentials for W and Ta isotopes were instrumental to achieve a very good description of the available microscopic cross section database. Model covariance data were generated with the Monte Carlo technique to produce a prior estimate for the covariance matrix. Experimental data were introduced through the GANDR system. The evaluated files were tested on selected fusion neutronics benchmarks and showed marked improvement compared to other existing evaluations.
\end{abstract}

\section{Introduction}

New design concepts of nuclear energy production and transmutation of radioactive waste are being investigated worldwide. Accurate nuclear data for tungsten isotopes are required because tungsten is a candidate material for first-wall components in fusion devices, target material for systems based on high-current accelerators, as well as neutron dosimetry with the ${ }^{186} \mathrm{~W}(\mathrm{n}, \gamma)$ reaction.

In spite of some recent attempts to improve the evaluated cross section data for tungsten [1], the status of the data is unsatisfactory. Systematic discrepancies are observed in criticality safety benchmarks containing tungsten [2], fusion neutronics benchmarks [3], and measured constants for neutron activation [4]. These discrepancies motivated the work presented herein. Preliminary results of experimental data analysis and evaluations for neutron interactions on tungsten isotopes ${ }^{180,182,183,184,186} \mathrm{~W}$ in the neutron energy range up to $60 \mathrm{MeV}$ are described.

\section{Resonance range}

The review of the resonance parameters of tungsten isotopes showed that no significant improvement in quality is possible without new measurements. Minor adjustments to existing resonance parameters of ${ }^{182} \mathrm{~W}$ were made. The sources of other resonance parameters were: Mughabghab [5] for ${ }^{180} \mathrm{~W}$, IRDF2002 [6] for ${ }^{186} \mathrm{~W}$ and ENDF/B-VII [7] for ${ }^{183} \mathrm{~W}$ and ${ }^{184} \mathrm{~W}$ isotopes. The unresolved resonance parameters were flagged for the calculation of self-shielding only; the cross

${ }^{a}$ Presenting author, e-mail: Andrej.Trkov@ijs.si sections from the model calculations in the URR were adopted as described below.

\section{Fast energy range}

Evaluations in the fast energy range were fully based on nuclear model calculations using the EMPIRE-2.19 code [8]. Starting values for nuclear model parameters were taken from RIPL-2 [9]. A crucial point was the selection of the coupledchannel optical model potential (OMP). Direct interaction cross sections to low-lying levels and transmission coefficients for the incident channel on ${ }^{180-186} \mathrm{~W}$ nuclei were obtained from the isospin-dependent dispersive coupled-channel OMP $[10,11]$. The same potential was used to calculate direct excitation of the collective levels in the continuum by DWBA method. Pre-equilibrium emission was considered using onecomponent exciton model PCROSS, which includes nucleon, gamma and cluster emission. Hauser-Feshbach and HofmannRichert-Tepel-Weidenmuller versions of the statistical model were used for the compound nucleus cross section calculations. Both approaches account for the multiple-particle emission, and the full gamma-cascade. Level densities were described by the "EMPIRE specific" formalism, which uses the super-fluid model below critical excitation energy and the Fermi gas model above. Deformation-dependent collective effects on the level densities due to nuclear vibration and rotation and their temperature-dependent damping were also taken into account Modified Lorenzian (MLO) radiativestrength function was taken as recommended by Plujko [9] and resulted in excellent agreement with the experimental neutron capture database. Total, capture, emission cross sections of neutrons and charged particles, average resonance parameters and angular distributions of neutron and proton scattering 


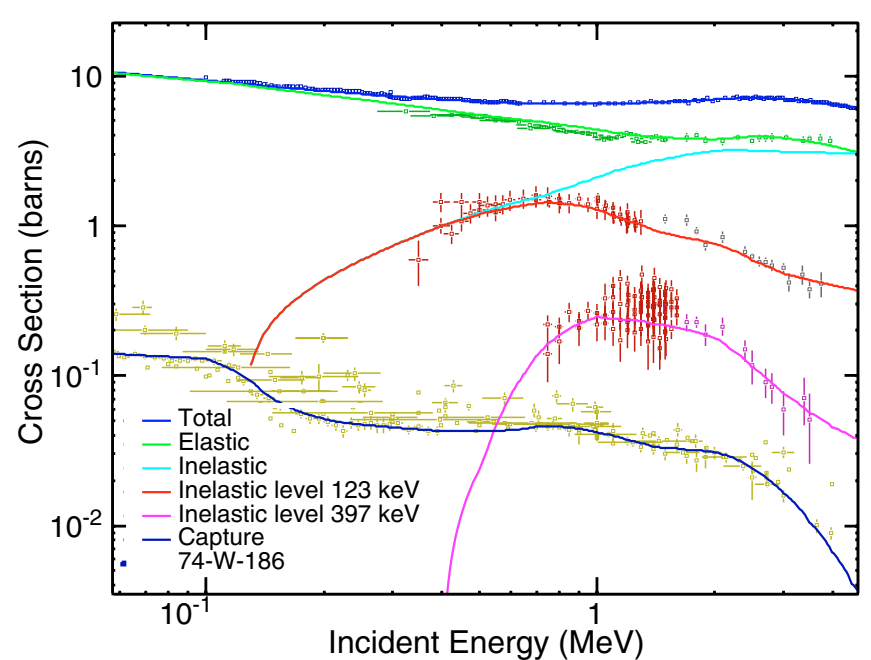

Fig. 1. Evaluated cross sections of ${ }^{186} \mathrm{~W}$ below $5 \mathrm{MeV}$.

on all tungsten isotopes were in good agreement with the available experimental data. An example of the evaluated cross sections for selected reactions of ${ }^{186} \mathrm{~W}$ below $5 \mathrm{MeV}$ in comparison with experimental data is shown in figure 1 .

\section{Covariance data}

The covariance matrix prior was generated by the Monte Carlo (MC) technique with the EMPIRE code. The prior and selected experimental data from the EXFOR database were fed into the GANDR system [12] to produce the final covariance matrices. An example of the prior covariance matrix generated by the MC method using nuclear model calculations for the $(\mathrm{n}, \gamma)$ cross section is shown in figure 2 .

\section{Validation}

To validate the data a series of benchmark test cases from the SINBAD compilation [13] were analysed:

- FNG Tungsten [14]: several reaction rates with sensitivities covering fast and intermediate energy ranges.

- FNS Clean Experiment on Tungsten [15]: neutron spectra above $5 \mathrm{keV}$ and neutron reaction rate measurements.

- OKTAVIAN tungsten sphere [16]: neutron spectra above $\sim 0.1 \mathrm{MeV}$ and gamma spectra measurements.

The benchmarks were analysed in detail using MC and deterministic transport codes, as well as codes for the cross section sensitivity and uncertainty studies [17]. They test major tungsten cross sections, particularly the (n,2n), (n, $\gamma)$, inelastic and elastic reactions. The cross section libraries ENDF/B-VII, JENDL-3.3 and fusion library FENDL-2.0 were of primary interest for comparison. The JEFF-3.1 library contains tungsten data from JENDL-3.3, therefore it is excluded; the FENDL-2.1 fusion library contains tungsten data from ENDF/B-VI Release 8, which differ from ENDF/BVII only in some minor details that do not affect the results, therefore either FENDL-2.1 or ENDF/B-VII results are shown. For the sake of completeness the comparison with a candidate evaluation for JEFF-3.2 library [18] is also given.

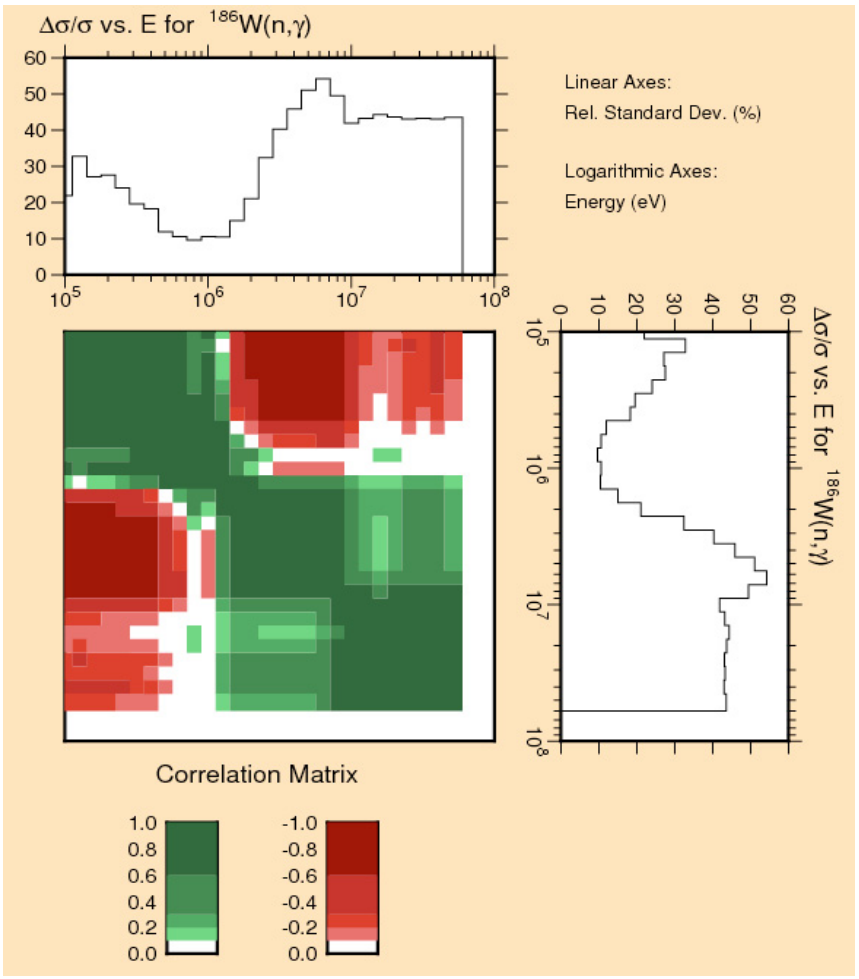

Fig. 2. Prior for the capture covariance matrix of ${ }^{186} \mathrm{~W}$.

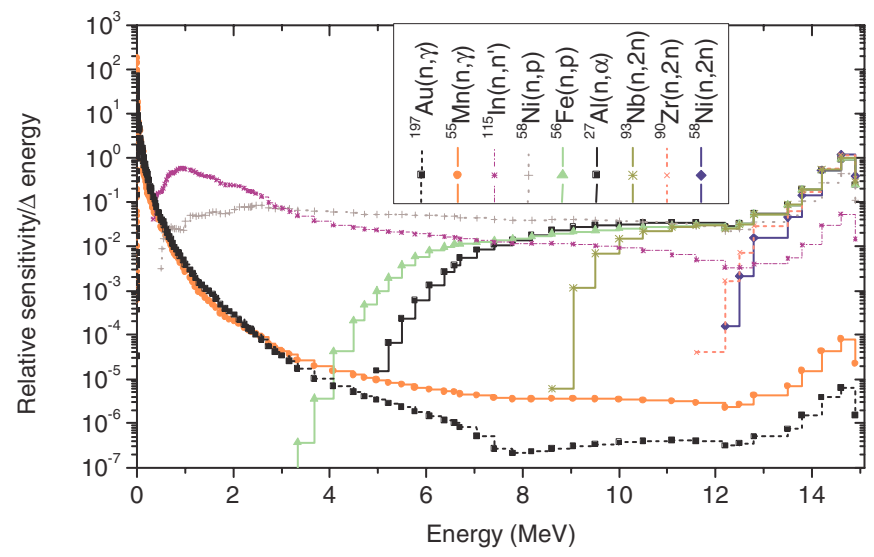

Fig. 3. Sensitivity with respect to the detector response functions at $35 \mathrm{~cm}$ in the tungsten block.

\subsection{FNG Tungsten experiment}

In the FNG Tungsten benchmark [14] the neutron reaction rates and gamma heating were measured at four positions along the central beam axis of the tungsten block, at approximately $5,15,25$ and $35 \mathrm{~cm}$ depths. The following nine reaction rates, covering fast and thermal neutron energies, were used in this exercise: ${ }^{27} \mathrm{Al}(\mathrm{n}, \alpha),{ }^{55} \mathrm{Mn}(\mathrm{n}, \gamma),{ }^{56} \mathrm{Fe}(\mathrm{n}, \mathrm{p}),{ }^{58} \mathrm{Ni}(\mathrm{n}, 2 \mathrm{n})$, ${ }^{58} \mathrm{Ni}(\mathrm{n}, \mathrm{p}),{ }^{90} \mathrm{Zr}(\mathrm{n}, 2 \mathrm{n}),{ }^{93} \mathrm{Nb}(\mathrm{n}, 2 \mathrm{n}),{ }^{115} \mathrm{In}\left(\mathrm{n}, \mathrm{n}^{\prime}\right)$, and ${ }^{197} \mathrm{Au}(\mathrm{n}, \gamma)$. The uncertainties in the ${ }^{55} \mathrm{Mn}(\mathrm{n}, \gamma)$, dosimetry cross sections are large, therefore this reaction was excluded from further consideration. The comparison of reaction rates for the other monitors from benchmark calculations with different libraries is shown in figure 4. 

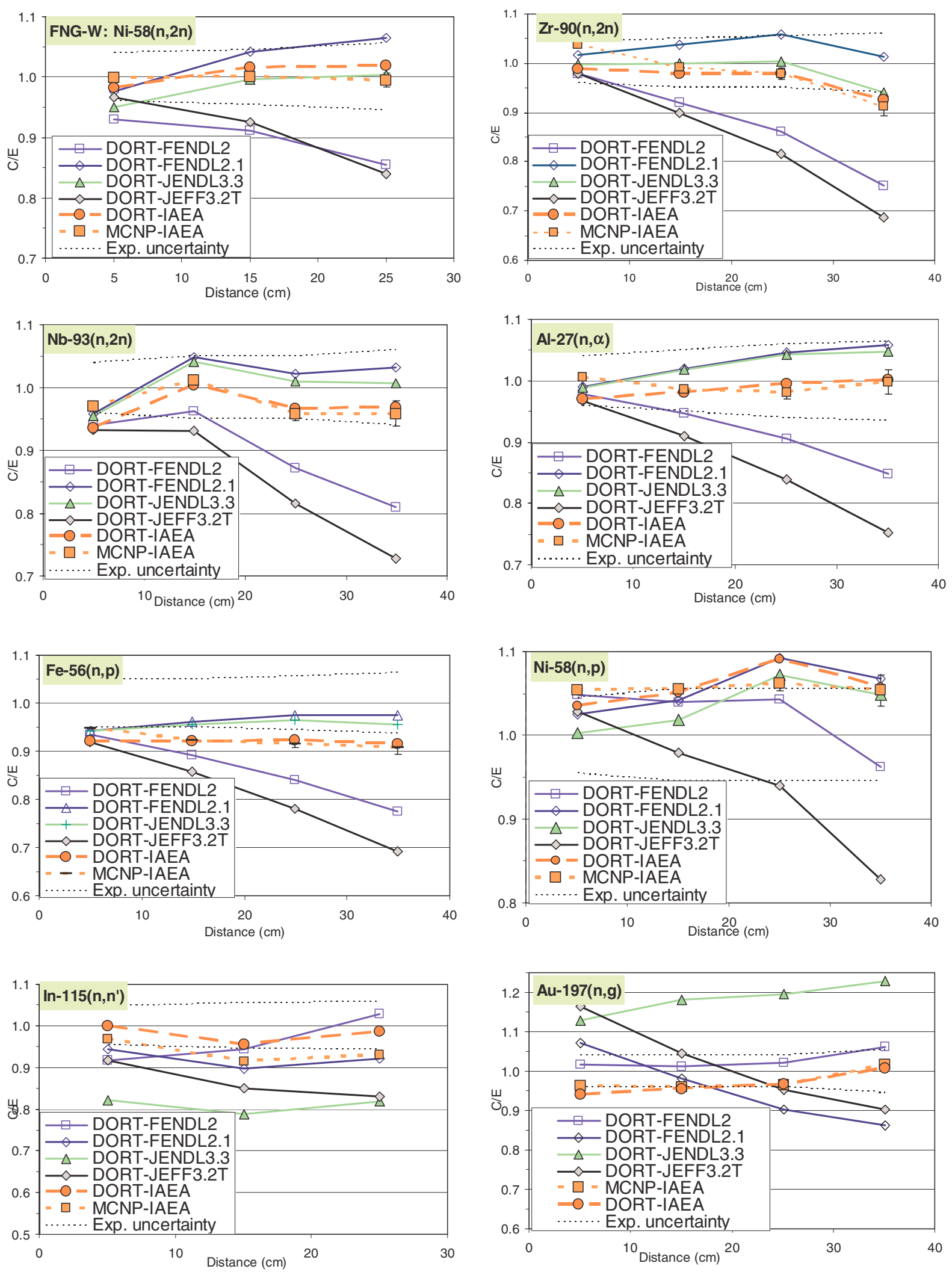

Fig. 4. Predicted reaction rates in the FNG benchmark. 


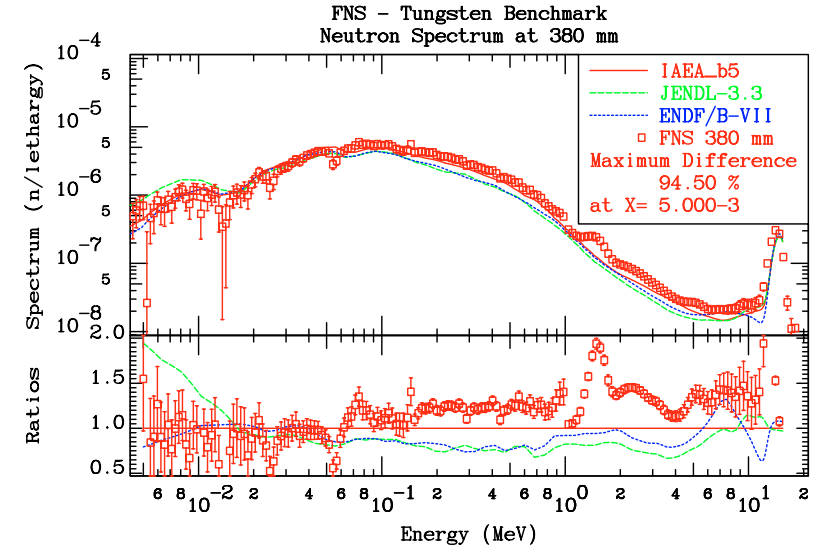

Fig. 5. Neutron spectra from the FNS benchmark.

\subsection{FNS clean experiment on tungsten}

The benchmark represents neutron flux attenuation from a deuterium-tritium source through a cylindrical assembly of about $40 \mathrm{~cm}$ thickness. The calculated neutron spectra at $38 \mathrm{~cm}$ penetration are compared to the measured ones in figure 5. Calculations with the new evaluation labelled IAEA_b6 indicate a marked improvement compared to other data libraries (JEF-3.2 corresponds to the evaluation of the ref. [18]). The spectra at smaller penetration depths show similarly good agreement.

\subsection{Oktavian tungsten sphere}

The benchmark represents neutron leakage spectrum from a tungsten sphere of $40 \mathrm{~cm}$ outer diameter and $10 \mathrm{~cm}$ shell thickness with a deuterium-tritium source at the centre. Qualitatively the results are very similar to those of the FNS benchmark, but due to the smaller thickness of the tungsten layer the benchmark is less sensitive to the differences in nuclear data libraries and hence less selective in discriminating between them.

\section{Conclusions}

Advanced nuclear model codes like EMPIRE with suitable model parameters prove to be capable of reproducing experimentally measured cross sections with good accuracy. They provide an excellent starting point for the evaluation of cross sections, double-differential data and their covariances. Using the results of model calculations as prior, experimental data and generalised least-squares codes can be used to improve the cross sections and constrain the uncertainties.

A trial evaluation of the tungsten isotopes was done. The results of a preliminary validation study on selected fusion neutronics benchmarks show significantly improved agreement with measurements. Activation measurements in the FNG benchmark, sensitive to a broad range of energies are particularly striking, since practically all results calculated with the new data lie within (or marginally outside) the uncertainty intervals.

The main shortcoming of the present file is the resonance energy range, where hardly any improvement is possible without new measurements.s
The authors are grateful to V. Semkova and A. Plompen for providing their unpublished charged-particle emission data, which proved crucial to guide our model calculations above $14 \mathrm{MeV}$.

\section{References}

1. P. Pereslavtsev et al., FZK and IIK, July 2006. Online at www.nea.fr/html/dbdata/projects/nds_eff_status.html.

2. S.C. van der Marck, Nucl. Data Sheets 107, 3061 (2006).

3. I. Kodeli, Reflection on the present status of tungsten cross sections based on the analysis of FNG and FNS Benchmark Experiments, EFFDOC-1002 (OECD/NEA, Paris, 2007).

4. I. Kodeli, A. Trkov, Validation of the IRDF-2002 Dosimetry Library, Nucl. Instrum. Meth. A (to be published).

5. S.F. Mughabghab, Atlas of Neutron Resonances (Elsevier, ISBN10:0-444-52035-X, 2006).

6. O. Bersillon, L.R. Greenwood, P.J. Griffin et al., International Reactor Dosimetry File 2002 (IRDF-2002), Technical Reports Series No. 452: (IAEA, Vienna, Austria, 2006). Online at wwwpub.iaea.org/MTCD/publications/PDF/TRS452_web.pdf.

7. M.B. Chadwick et al., Nucl. Data Sheets 107, 2931 (2006).

8. M. Herman, P. Obložinský, R. Capote, M. Sin, A. Trkov, A. Ventura, V. Zerkin, in Proceedings of the International Conference on Nuclear Data for Science and Technology, 27 Sept. - 1 Oct. 2004, Santa Fe, NM, USA.; AIP Conf. Proc. 769, 1184 (2005). Online at www.nndc.bnl.gov/empire219/

9. T. Belgya, O. Bersillon, R. Capote et al., Handbook for calculations of nuclear reaction data: Reference Input Parameter Library-2, Technical Report IAEA-TECDOC-1506 (IAEA, Vienna, Austria, 2006). Available online at wwwnds.iaea.org/RIPL-2/

10. R. Capote, E.Sh. Soukhovitski, J.M. Quesada, S. Chiba, Isospin dependent dispersive coupled channel optical model potential for tungsten isotopes, $11^{\text {th }}$ International Conf. on Nuclear Reaction Mechanisms, Varenna, Italy, June 12-16, 2006.

11. R. Capote, M. Sin, A. Trkov, NEMEA-3: $3^{\text {rd }}$ Workshop on Neutron Measurements, Evaluations and Applications, Oct. 2528, 2006 Borovets, Bulgaria (to be published by the European Commission).

12. D.W. Muir, Global Assessment of Nuclear Data Requirements - The GANDR Project, An IAEA Nuclear Data Section Data Development Project. Online at www-nds.iaea.org/gandr/.

13. I. Kodeli, E. Sartori, B. Kirk, SINBAD Shielding Benchmark Experiments Status and Planned Activities, in Proc. ANS $14^{\text {th }}$ Biennial Topical Meeting of the Radiation Protection and Shielding Division, Carlsbad, NM, USA, 3-6 April 2006. Online www.nea.fr/html/science/shielding/sinbad/sinbadis.htm

14. P. Batistoni, M. Angelone, L. Petrizzi, M. Pillon J. Nucl. Mat. 329-333, 683 (2004).

15. F. Maekawa, C. Konno, Y. Kasugai, Y. Oyama, Y. Ikeda, Data Collection of Fusion Neutronics Benchmark Experiment Conducted at FNS/JAERI, JAERI-Data/Code 98-021 (1998).

16. Sub Working Group of Fusion Reactor Physics Subcommittee: Collection of Experimental Data for Fusion Neutronics Benchmark, JAERI-M-94-014 (1994).

17. I. Kodeli, J. Nucl. Mat. 329-333, 717 (2004).

18. H. Vonach et al., Evaluation of the fast neutron cross sections of the $W$ and Ta including complete covariance information (these proceedings). 